\title{
RNAi-mediated knockdown of vascular endothelial growth factor inhibits vascularization and tumor growth in renal cell carcinoma
}

\author{
MENG GU*, KE ZHANG* , HAI-JUN YAO, JUAN ZHOU, YU-BING PENG, MING-XI XU and ZHONG WANG \\ Department of Urology, Shanghai Ninth People's Hospital, Shanghai Jiao Tong University \\ School of Medicine, Shanghai 200011, P.R. China
}

Received April 1, 2015; Accepted July 27, 2015

DOI: $10.3892 /$ ijmm.2015.2326

\begin{abstract}
In the present study, we aimed to examine the effects of the knockdown of vascular endothelial growth factor (VEGF) by RNA interference (RNAi) on vascularization and tumor growth in renal cell carcinoma (RCC). For this purpose, a lentiviral vector expressing VEGF-shRNA was constructed and transfected into 293T cells. The efficiency of RNAi was determined by infecting human 786-O RCC cells with viral particles and measuring the VEGF mRNA levels by reverse transcription-quantitative polymerase chain reaction (RT-qPCR). The effect of transfection with VEGF-shRNA on the secreted VEGF levels was also examined and the inhibitory effects on vascularization were also examined using a chick chorioallantoic membrane (CAM) assay. An RCC xenograft model was established in nude mice by implanting 786-O cells to form subcutaneous tumors. VEGF expression was observed by immunohistochemical (IHC) staining of the xenograft tumors. The tumor volume and tumor inhibition rate were also recorded. The apoptosis of the cancer cells was measured by TUNEL assay and the efficiency of tumor inhibition was estimated. The interference rate of VEGF-shRNA was $72.2 \%$ in the 786-O cells. Our results revealed that VEGF mRNA expression, the secreted VEGF level in the 786-O cells and the total vessel length were markedly reduced in the VEGF-shRNA-transfected cells compared with the controls (all $\mathrm{P}<0.05$ ). Compared with the controls, injections of lentivirus expressing VEGF-shRNA significantly inhibited tumor growth, and reduced tumor mass and VEGF expres-
\end{abstract}

Correspondence to: Dr Zhong Wang or Dr Hai-Jun Yao, Department of Urology, Shanghai Jiao Tong University School of Medicine, Shanghai 200011, 639 Zhi Zaoju Road, Huangpu Area, Shanghai 200120, P.R. China

E-mail: zhongwang2000@sina.com

E-mail: dryaohj@163.com

*Co-first authorship

Key words: vascular endothelial growth factor, lentiviral vectors, renal cell carcinoma, chick chorioallantoic membrane assay, xenograft tumor, nude mice, gene silencing, gene therapy sion in the tumor tissue (all $\mathrm{P}<0.05$ ). The apoptotic index in the treatment group was significantly higher than that in the controls (both $\mathrm{P}<0.05$ ). Thus, our data indicate that the inhibition of VEGF expression by RNAi reduces VEGF mRNA levels, and inhibits angiogenesis and tumor growth in RCC, providing a future treatment option for RCC.

\section{Introduction}

Renal cell carcinoma (RCC) is the most common type of kidney cancer in adults and accounts for $80-95 \%$ of kidney cancer cases (1). RCC is asymptomatic during the early stages of the disease; thus, it is only diagnosed at a late stage and a high proportion of patients with RCC present with distant metastases at diagnosis (2). The treatment options for metastatic RCC are extremely limited due to the inherent resistance of these types of tumors to chemotherapy, radiotherapy and other systemic therapies (3). Immunotherapeutic agents, such as interleukin (IL)-2 and interferon- $\alpha$ (IFN- $\alpha$ ) have gained considerable attention in recent years in an aim to control tumor growth and metastatic progression in RCC (4). However, only a very limited subset of patients with RCC benefit from cytokine therapy, with modest objective response rates (5). Therefore, innovative and effective therapies for metastatic RCC are urgently required and new treatment strategies for RCC are being explored. RCC progression is intimately associated with tumor angiogenesis and the upregulation of vascular endothelial growth factor (VEGF) expression (6). The human $V E G F$ gene localizes to chromosome $6 \mathrm{p} 12$ and consists of 8 exons (7). In RCC, the increased transcription of the VEGF gene and the increased levels of secreted VEGF correspond to the increased tumor size and changes in tumor behavior are observed (8).

VEGF is one of the most important endothelial cell-specific angiogenic factors. It plays crucial roles in tumor angiogenesis through its specific effects on vascular endothelial cells, including the promotion of cell mitogenesis, cell migration and lumen formation, leading to a powerful angiogenic response (9). The specificity of the effects of VEGF is achieved by the preferential expression of its two receptors, namely VEGF-R1 (FLT-1) and VEGF-R2 (KDR), on VEGF target cells and the expression of these receptors is observed in tumor-associated endothelial cells (10). Therefore, VEGF is a promising and a high-value target for the inhibition of tumor angiogenesis in growing tumors. In this study, in order to 
explore novel therapeutic strategies for the treatment of RCC, we used a mouse xenograft model, a favorable animal model for evaluating new therapeutic approaches (11). In a mouse xenograft model, subcutaneous injections of human tumor cells, in this case RCC cells, result in the establishment of primary tumors at the sites of injection, and the development of subsequent metastases as the tumor progresses, and these events mimic the behavior of human tumors in vivo (12). We developed RNA interference (RNAi) tools to silence VEGF expression in human RCC cells in vitro, as well as in an in vivo xenograft model. RNAi, is a sequence-specific post-transcriptional gene silencing technique mediated by small interfering RNAs (siRNAs) homologous to the silenced gene (13) or by short hairpin RNA (shRNA) expression vectors (14). In the present study, we used the lentivirus-based RNAi strategy as a tool against RCC, in order to assess the effects of the knockdown of VEGF by RNAi on vascularization and tumor growth in RCC.

\section{Materials and methods}

Ethics statement. Animal experiments were conducted in strict accordance with the approved animal protocols and guidelines established by the Medicine Ethics Review Committee for animal experiments of the First Affiliated Hospital of China Medical University. All efforts were made to minimize the suffering of the animals.

Construction and identification of vectors. Based on the human VEGF mRNA sequence (NCBI GenBank, gene number: AF022375) (15), the following oligonucleotides were designed with BLOCK-iT ${ }^{\mathrm{TM}}$ RNAi Designer (Invitrogen Life Technologies, Carlsbad, CA, USA): interference sequence, 200-F, TGCTGTGAAGATGTACTCGATCTCATGTTTT GGCCACTGACTGACATGAGATCGTACATCTTCA and 200-R, TGCTGTGAAGATGTACTCGATCTCATGTTTT GGCCACTGACATGAGAT CGTACATCTTCA; and negative control, Negative-F, TGCTGAAATGTACTGCGCGTGGA GACGTTTTGGCCACTGACTGACGTCTCCACGCAGTA CATTT and Negative-R, CCTGAAATGTACTGCGTGGAG ACGTCAGTCAGTGGCCAAAACGTCTCCACGCGCAGTA CATTTC. Negative-F and Negative-R, as well as 200-F and $200-\mathrm{R}$ were mixed in equal amounts (500 pmol each), heated for $10 \mathrm{~min}$ at $89^{\circ} \mathrm{C}$, gradually cooled to $65^{\circ} \mathrm{C}$ in annealing buffer (Tris- $\mathrm{HCl} 50 \mathrm{mM}, \mathrm{MgCl}_{2} 10 \mathrm{mM}$, DTT $10 \mathrm{mM}$, ATP $1 \mathrm{mM}$, BSA $25 \mu \mathrm{g} / \mathrm{ml}$,DMSO $10 \%$ ) to generate double-stranded DNA. The double-stranded DNA was cloned into the plasmid vector, plenti6.3-miR (Novobio, Shanghai, China). Positive clones were sequence-verified.

Construction of VEGF-shRNA expression vector. To effectively inhibit VEGF expression, it was necessary to design a shRNA expresssion vector that was capable of downregulating VEGF isoforms. Therefore, we conducted a bioinformatics analysis and selected a sequence that was conserved in all VEGF isoforms as the target for RNAi.

Lentiviral packaging. The 239T cells (Novo Biochemical Industries, Inc.) a the logarithmic growth phase were trypsinized and counted. The cells were seeded in $10-\mathrm{cm}$ petri dishes at a density of $6 \times 10^{6}$ cells/dish. The cell culture medium was replaced with Opti-MEM medium prior to transfection. Packaging mix $(9 \mu \mathrm{g})$ and lentiviral plasmid $(3 \mu \mathrm{g})$ were added into $1.5 \mathrm{ml} \mathrm{Opti-MEM}$ (prewarmed to $37^{\circ} \mathrm{C}$ ) and gently mixed. Lipofectamine $2000(36 \mu \mathrm{l})$ was added to another $1.5 \mathrm{ml}$ Opti-MEM and gently mixed. The plasmid solution and Lipofectamine 2000 diluent were combined to form complexes. Subsequently, $3 \mathrm{ml}$ of the plasmid/liposome complex $(3 \mathrm{ml})$ were added to the petri dishes, evenly mixed, and incubated in a $5 \% \mathrm{CO}_{2}$ incubator at $37^{\circ} \mathrm{C}$ for $6 \mathrm{~h}$. The culture medium was replaced with complete medium (DMEM + 10\% (FBS; Biowest Co., Nuaillé, France). After 48 h, the cell culture supernatant was collected, centrifuged at 3,000 rpm for $10 \mathrm{~min}$, and filtered through $0.45-\mu \mathrm{m}$ filters. The virals particles were purified by ultracentrifugation $(50,000 \times \mathrm{g}$ for $2 \mathrm{~h})$, resuspended in $200 \mu \mathrm{l}$ DMEM (HyClone Co., Logan, UT, USA), and stored at $-80^{\circ} \mathrm{C}$ until further use.

Titer determination. Viral titers were determined by infecting 293 T cells. The cells were seeded in 96-well plates at a density of $8 \times 10^{3}$ cells $(100 \mu \mathrm{l}) /$ well each day prior to infection. Vector stocks were gradient diluted in the presence of $8 \mu \mathrm{g} / \mathrm{ml}$ of Polybrene (Sigma-Aldrich, St. Louis, MO, USA) at $1 \times 10^{-3}$ to $1 \times 10^{-8} \mathrm{ml}$ per $50 \mathrm{ml}$. After the original medium was removed, the $50 \mu \mathrm{l} /$ well of the diluted medium containing lentiviral vector particles were added and mixed, and another $50 \mu$ l diluted lentiviral medium was added to each well, with 3 replicates at each dilution. After a 48-h incubation in a $\mathrm{CO}_{2}$ incubator, $100 \mu \mathrm{l}$ fresh medium were added to each well. After 5-6 days, the number of green fluorescent protein (GFP)-positive cells was scored by fluorescence microscopy and/or fluorescence-activated cell sorting analysis to quantify the titer. The number of fluorescent cells decreased with dilution. The number of fluorescent cells in the well with the largest dilution multiple was counted. Viral titer $(\mathrm{TU} / \mathrm{ml})=($ the number of fluorescent cells $\mathrm{x}$ the number of transfected cells $/ 100 \mathrm{x}$ the volume of added diluted lentiviral medium of each well) $\mathrm{x} 1$ /dilute strength (concentration of diluent).

Cell culture and lentiviral infection. The human RCC cell line, 786-O, was purchased from the Cell Bank of Shanghai Institute of Cell Biology, Chinese Academy of Sciences (Shanghai, China). The cells were maintained in RPMI-1640 medium supplemented with FBS (Gibco Ltd., Grand Island, NY, USA), and incubated in $5 \% \mathrm{CO}_{2}$ at $37^{\circ} \mathrm{C}$. The transient viral transfection of human RCC 786-O cells was conducted using $1 \mu 1$ Polybrene and lentivirus at a multiplicity of infection (MOI) of 100. The cells were divided into 3 groups: the blank control group (no viral transfection); the negative control group (cells transfected with LV-NC-shRNA); and the VEGF-shRNA group (cells transfected with LV-VEGF-shRNA).

Reverse transcription quantitative fluorescence polymerase chain reaction ( $R T-q P C R$ ) of VEGF $m R N A$ expression. To quantify VEGF mRNA expression in the 786-O-transfected cells, RNA was extracted using TRIzol ${ }^{\circledR}$ reagent (Invitrogen Life Technologies), and reverse transcription was conducted using the Reverse Transcription kit (Bio-Rad Laboratories, Hercules, CA, USA) following the manufacturer's instructions at $48 \mathrm{~h}$ after transfection. The primers used for PCR were as 
follows: VEGF upstream, 5'-ACTGCCATCCAATCGAG ACCC-3' and downstream, 5'-TGAGGTTTGATCCGCAT AATC-3'; and the internal reference, $\beta$-actin upstream, 5'-ACTCTTCCAGCCTTCCTTCC-3' and downstream, 5'-GTACTTGCGCTCAGGAGGAG-3'. The reaction system was prepared with a Fluorescence Real-time PCR kit (Bio-Rad Laboratories) following the manufacturer's instructions. The reaction was carried out under following conditions: 40 cycles of predenaturation at $95^{\circ} \mathrm{C}$ for $120 \mathrm{sec}$, denaturation at $95^{\circ} \mathrm{C}$ for $10 \mathrm{sec}$, annealing at $60^{\circ} \mathrm{C}$ for $30 \mathrm{sec}$, and extension at $70^{\circ} \mathrm{C}$ for $45 \mathrm{sec}$, followed by the real-time detection of fluorescent signals. The data analysis for qPCR was relatively calculated using the $2^{-\Delta \Delta C T}$ method [cycle threshold $(C T)$ ]. Interference efficiency $=\left(1-2^{-\Delta \Delta C \mathrm{CT}}\right) \times 100$.

Chick chorioallantoic membrane (CAM) assay. Cell culture supernatants obtained from the 786-O cells cultured for $72 \mathrm{~h}$ were lyophilized and dissolved in a total volume of RPMI-1640 (100 $\mu$ l). The corresponding cell count after $72 \mathrm{~h}$ of culture was $5 \times 10^{7}$. Similarly, culture supernatants from 786-O cells transfected with the shRNAs were also collected and processed. The concentration of VEGF in the cell culture supernatants was estimated using an enzyme-linked immunosorbent assay (ELISA) kit (Jingmei Biotech Co. Ltd., Shenzhen, China). These supernatants were also used for CAM assays as described below. White fertilized eggs (Zhejiang Tianyuan Bio-Pharmaceutical Co., Ltd., Hangzhou, China), incubated for 9 days, were selected. The air chamber of the eggs was opened to expose the chorioallantoic membrane. On the 10th day of incubation, the eggs were randomly divided into 4 groups of $10 \mathrm{eggs} / \mathrm{group}$ as follows: a volume of $100 \mu \mathrm{l}$ RPMI-1640 medium was added between the omphalomesenteric veins and the chick embryo chorioallantoic membrane using a micropipette as a blank control. The other 3 groups were: culture supernatant of uninfected 786-O cells (positive control group); culture supernatant of 786-O cells infected with lentivirus LV-VEGF-shRNA (VEGF-shRNA group); and the culture supernatant of 786-O cells infected with lentivirus LV-NC-shRNA (negative control group). All the eggs were then incubated at $37^{\circ} \mathrm{C}$ for $48 \mathrm{~h}$. Fixation was carried out in formaldehyde and acetone (1:1)on the 12th day. The chick embryo chorioallantoic membrane was cut off, dried and photographed under an optical microscope (DSX510; Olympus, Tokyo, Japan; x10 magnification),GIF-2T240; Olympus, Japan), selecting 6 random fields. Using an imageprocessing system (Gene Co. Ltd, USA), the vascular branches of the first and second level in the chorioallantoic membrane within the field were traced, the vascular branch points were counted manually, and the relative total vessel length was measured. The mean value of the 6 fields was calculated. The number of vascular branches and total vessel length (vascular density index) of all the groups were compared.

Establishment of RCC xenograft model in nude mice. Four-week-old female BALB-C nude mice $(\mathrm{n}=36$; weighing approximately $20 \mathrm{~g}$ ) were purchased from Shanghai Silaike Experimental Animal Co., Ltd., Chinese Academy of Medical Sciences (Shanghai, China). All BALB-C nude mice were kept under specific pathogen-free (SPF) conditions in the Experimental Animal Center of the Chinese Academy of
Medical Sciences. A total of 18 nude mice were randomly selected and divided into 3 groups (blank control, negative control and VEGF-shRNA group) of 6 mice/group. The cells in the logarithmic growth phase were collected from the blank control group, negative control group and VEGF-shRNA group. To establish a RCC xenograft model, the nude mice in the 3 groups were injected subcutaneously into the lower back with $5 \times 10^{6}$ cells suspended in $0.2 \mathrm{ml}$ of phosphate-buffered saline (PBS). Tumor occurrence and growth in each group was observed and recorded. Tumor volume was determined by external measurements using a caliper and calculated as $\mathrm{V}=\mathrm{LW}^{2} / 2$, where $\mathrm{L}$ and $\mathrm{W}$ represented the larger and the smaller tumor diameter, respectively. The observation period ended at 30 days from the day of injection. At this time point, the nude mice were sacrificed by cervical dislocation and the tumors were removed, weighed using an electronic balance (JA-5003 Electronic Precision Balance, Shanghai, China), formalin-fixed, paraffin-embedded and sliced using a paraffin slicing machine ( Leica RM2135 microtome, Lecia, Germany). Immunohistochemical (IHC) staining for VEGF expression was then performed as described below.

Immunohistochemistry. The formalin-fixed paraffin-embedded slices were subjected to immunohistochemical staining for VEGF expression. The tumor slices were deparaffinized, rehydrated in $3 \% \mathrm{H}_{2} \mathrm{O}_{2}$ for $10 \mathrm{~min}$ and endogenous peroxidase was quenched. The slides were then were washed with clear water twice, followed by the addition of citric buffer, and heating in a microwave at middle power for $3 \mathrm{~min}$. After being cooled to the room temperature, the slices were washed with clear water twice; the glass slides were then soaked in PBS for $5 \mathrm{~min}$, washed twice, and serum was added at a 1:10 dilution (900 $\mu \mathrm{l}$ PBS:100 $\mu 1$ serum blocking solution), followed by incubation at $37^{\circ} \mathrm{C}$ for $30 \mathrm{~min}$. Subsequently, a rabbit anti-human primary antibody specific for VEGF (Sigma-Aldrich) was added followed by overnight incubation at $4^{\circ} \mathrm{C}$ in a refrigerator. The same rabbit anti-human without specificity $\operatorname{IgG}$ was used as the primary antibody in the negative control group. All slides were subsequently washed with PBS 3 times for 5 min each time, and incubated with rabbit anti-goat secondary antibody (Sigma-Aldrich) for $30 \mathrm{~min}$ at $37^{\circ} \mathrm{C}$. The slices were then washed with PBS 3 times for 5 min each time, and incubated with a 1:100 dilution of streptavidin-biotin complex (SABC; $10 \mu 1 \mathrm{SABC}: 990 \mu 1 \mathrm{PBS}$ ) for $30 \mathrm{~min}$ at $37^{\circ} \mathrm{C}$. The slices were stained with $\mathrm{DAB}$, counterstained with hematoxylin after being washed, and then mounted with neutral gum. Observation was performed under a high power microscope (x400 magnification), and the VEGF-positive cells were those with a distribution of brown granules in the cytoplasm and/or nucleus. Immunoreactive slices were quantitatively analyzed with 5 intratumoral fields (approximately 100 cells) selected from each slice. The positive VEGF rate (from automatic computer analysis) $=$ the area of positive cells/the total area of negative cells, the mean value was obtained as the positive VEGF rate (\%). The results were determined using the double blind method (the pathologists and specimen collectors were unaware of any information regarding the specimens).

Treatment of tumor-bearing nude mice. A total of 18 nude mice were used for the xenograft experiments and each mouse was 
Table I. Interference efficiency of RNAi lentiviral plenti6.3-miR-200 targeting vascular endothelial growth factor (VEGF) detected by RT-qPCR (\%).

\begin{tabular}{lcccc}
\hline Group & $\Delta \mathrm{CT}$ & $\Delta \Delta \mathrm{CT}$ & $2^{-\Delta \Delta \mathrm{CT}}$ & Interference rate \\
\hline VEGF-shRNA group & 10.652100 & 1.847 & 0.27797 & 72.2 \\
Negative control group & 8.412102 & -0.393 & 1.31312 & 31.3 \\
Blank control group & 8.805073 & 0 & 1 & 0 \\
\hline
\end{tabular}

VEGF-shRNA group, cells transfected with LV-VEGF-shRNA; negative control group, cells transfected with LV-NC-shRNA; blank control group, cells without viral transfection; CT, cycle threshold; interference efficiency $=\left(1-2^{-\Delta \Delta C T}\right) \times 100$.

injected subcutaneously with a cell suspension $(20 \mu \mathrm{l})$. The size of each subcutaneous mouse tumor was measured daily using a Vernier caliper. A diameter $\geq 5 \mathrm{~mm}$ was considered as positive for a tumor. The mice were randomly divided into 3 groups with 6 mice/group as follows: mice intratumorally injected with $0.1 \mathrm{ml}$ LV-VEGF-shRNA/mouse/time (treatment group); mice intratumorally injected with $0.1 \mathrm{mlLV}-\mathrm{NC}$-shRNA/mouse/time (negative control group); and mice intratumorally injected with $0.1 \mathrm{ml} \mathrm{PBS} /$ mouse/time (blank control group). Treatment was carried out using an intratumoral multi-point injection every 4 days for a total of 5 times. After 20 days and following observation, the nude mice were sacrificed by cervical dislocation and the tumors were weighed, formalin-fixed, paraffin-embedded, and sliced for apoptosis detection (apoptosis assay). The tumor growth curve was drawn and the the tumor inhibition rate was calculated. Tumor inhibition rate $=$ (tumor volume in control group - tumor volume in experimental group)/tumor volume in control group x100.

TUNEL assay. The apoptosis was measured by TUNEL assay using an In Situ Cell Death Detection kit, (Roche Applied Sicence, Pleasanton, CA, USA) following the manufacturer's instructions. Positive cells are regarded as those with a tawny nucleus, nuclear chromatin condensation and an irregular cell shape. Negative cells are regarded as those without any changes in cell shape or coloration, or with only a slight coloration. Under a microscope, quantitative analysis was performed by counting the number of positive cells per high power microscopic field; for each sample, 5 high power microscopic fields (200 cells) were counted. The number of positive cells was presented as a percentage, and regarded as the apoptotic index (AI).

Statistical analysis. All statistical analyses were performed using SPSS 17.0 software (SPSS Inc., Chicago, IL, USA), and the data are presented as the means \pm standard deviation (SD). Statistical comparisons between 2 groups were conducted using the t-test or analysis of variance (ANOVA). P-values for all tests were two-tailed, and a $\mathrm{P}<0.05$ was considered to indicate a statistically significant difference.

\section{Results}

Results of sequencing analysis. The results from DNA sequencing analysis confirmed the successful cloning of the VEGF-shRNA into plenti6.3-miR (Fig. 1).
Viral titer and transfection efficiency. Twenty-four hours after transfection of the plenti6.3-miR-200 into 293T cells for packaging the lentiviral particles, geen fluorescence was detected in the majority of $293 \mathrm{~T}$ cells. The fluorescence microscopy image (x100 magnification) and the image of the same field of cells in visible light are shown in Fig. 2. Lentiviral plenti6.3-miR-200 transfected into the 293T cells was observed by fluorescence microscopy and ordinary light microscopy (x100 magnification), and it was detected that the number of GFP-expressing cells in the wells containing viral stock was 37, 25 and 35, respectively. Therefore, the viral titer was calculated to be $3.23 \times 10^{9} \mathrm{TU} / \mathrm{ml}$. These results suggested the successful transfection of $293 \mathrm{~T}$ cells.

Transfection of 786-O cells with plenti6.3-miR-200 inhibits VEGF expression. The results of 48-h transfection with lentivirus plenti6.3-miR-200 in 786-O cells were observed under a fluorescence microscope (Fig. 3). The infection rate of the RNAi lentivirus plenti6.3-miR-200 in the 786-O cells was calculated (infection rate $=$ the number of fluorescent cells/cell total number $\mathrm{x} 100$ ), and the result reached $90 \%$. VEGF expression was detected by RT-qPCR, as shown in Table I. The results of RT-qPCR revealed that the interference rate of RNAi lentivirus plenti6.3-miR-200 in the VEGF-shRNA group was $72.2 \%$.

Effect of VEGF-shRNA on vascularization in the chorioallantoic membrane. The comparison of VEGF levels in the cell culture supernatants and the extent of vascularization in the blank control, positive control, VEGF-shRNA and negative control groups are shown in Table II. The VEGF level in the culture medium without FBS (blank control group) was 0 . The VEGF expression level in the cell culture supernatant of the VEGF-shRNA group was markedly lower than that of the negative control and positive control groups $(196.63 \pm 56.28$ vs. $1231.10 \pm 121.86 \mu \mathrm{g} / \mathrm{ml}$ vs. $1241.08 \pm 126.64 \mu \mathrm{g} / \mathrm{ml}, \mathrm{P}<0.05)$. The vascular branch points in the VEGF-shRNA, negative control and positive control groups were significantly greater than those of the blank control group $(72.01 \pm 9.56$ vs. $74.21 \pm 8.91$ vs. $76.89 \pm 9.08$ vs. $49.65 \pm 6.72$, all $\mathrm{P}<0.05)$. The relative total vessel length in the VEGF-shRNA, negative control and positive control groups was significantly longer than that of the blank control group (all $\mathrm{P}<0.05$ ). Additionally, the relative total vessel length in the VEGF-shRNA group was significantly shorter than that of the negative control and positive control groups $(66.34 \pm 10.31$ vs. $86.08 \pm 15.53$ vs. $86.63 \pm 14.62$, all $\mathrm{P}<0.05)$. The vascular network structure in the chorioallantoic 


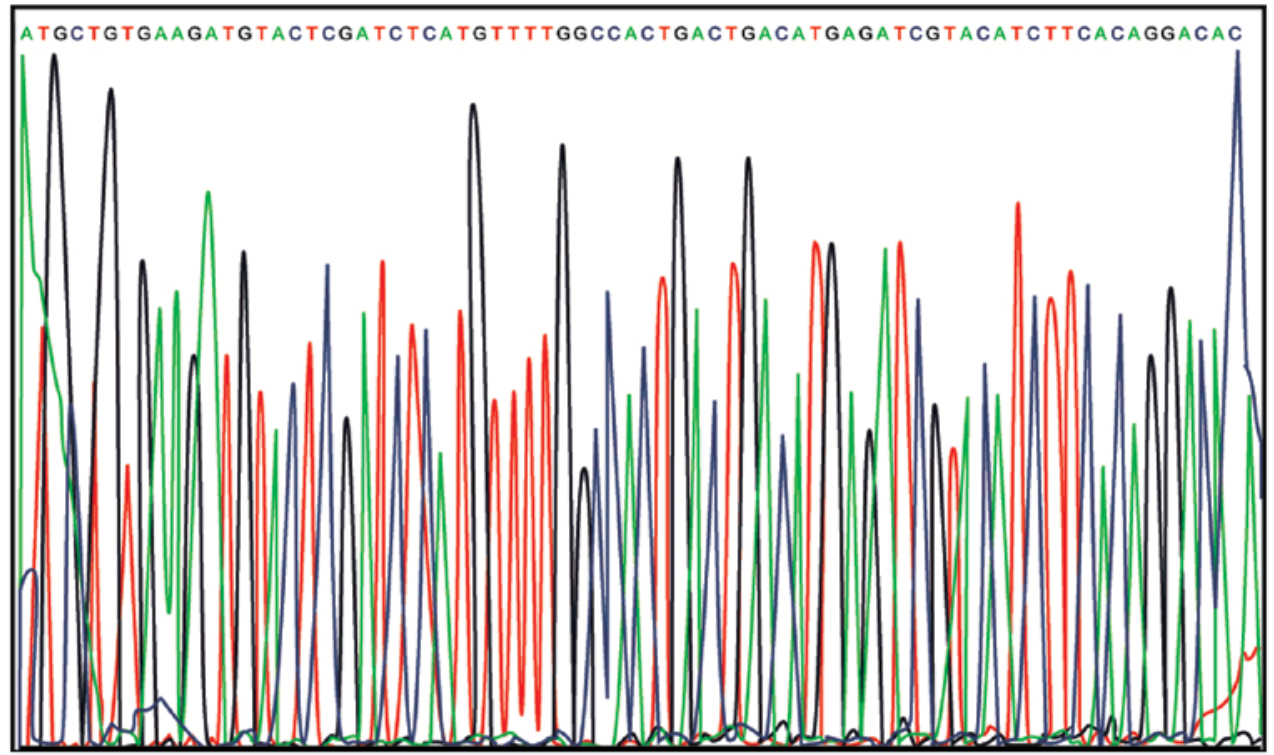

Figure 1. DNA sequencing results, by oligo cloning into plenti6.3-miR, showing that the obtained sequence was exactly in accordance with the expected sequence.
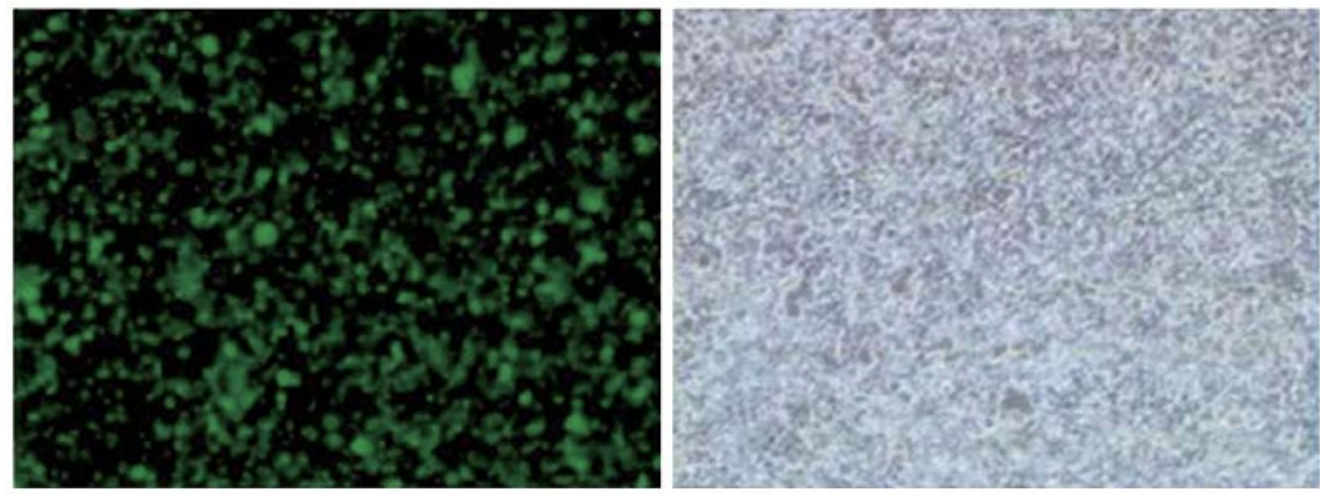

Figure 2. Observation of RNA interference (RNAi) lentiviral packaging under a fluorescence microscope (x100 magnification) showing green fluorescent protein (GFP) in the majority of $293 \mathrm{~T}$ cells within the same field of vision compared with an image of the same field using visible light. Left panel, a fluorescence image; right panel, a visible-light image.
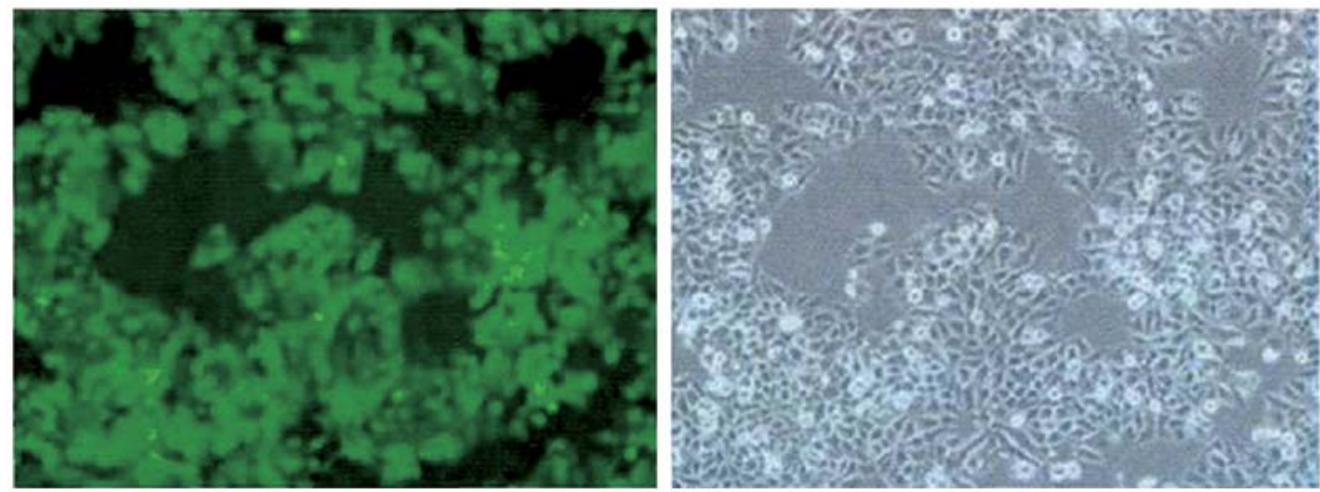

Figure 3. Transfection efficiency of 786-O cells $48 \mathrm{~h}$ after transfection with RNA interference lentiviral plenti6.3-miR-200 under a fluorescence microscope (x100 magnification). Left panel, a fluorescence image; right panel, a visible-light image.

membrane is presented in Fig. 4. Compared with the blank control group, the vascular branches in the chorioallantoic membrane of the VEGF-shRNA group increased slightly, and in the chorioallantoic membrane of the negative control and positive control groups, this increase was more visible with abnormal capillary network structures present. 
Table II. Comparison of the density of vascularization in chick embryo chorioallantoic membrane and vascular endothelial growth factor (VEGF) levels in the cell culture supernatant.

\begin{tabular}{lccc}
\hline Group & Vascular branch point & Relative total vessel length & VEGF level $(\mu \mathrm{g} / \mathrm{ml})$ \\
\hline Blank control group & $49.65 \pm 6.72$ & $43.55 \pm 7.45$ & 0 \\
VEGF-shRNA group & $72.01 \pm 9.56^{\mathrm{a}}$ & $66.34 \pm 10.31^{\mathrm{a}-\mathrm{c}}$ & $196.63 \pm 56.28^{\mathrm{a}-\mathrm{c}}$ \\
Negative control group & $74.21 \pm 8.91^{\mathrm{a}}$ & $86.08 \pm 15.53^{\mathrm{a}}$ & $1231.10 \pm 121.86^{\mathrm{a}}$ \\
Positive control group & $76.89 \pm 9.08^{\mathrm{a}}$ & $86.63 \pm 14.62^{\mathrm{a}}$ & $1241.08 \pm 126.64^{\mathrm{a}}$ \\
F-value & 21.01 & 26.95 & 514.4 \\
P-value & $<0.001$ & $<0.001$ & $<0.001$ \\
\hline
\end{tabular}

${ }^{\mathrm{a} C}$ Compared with blank control group, $\mathrm{P}<0.05$. ${ }^{\mathrm{b}}$ Compared with negative control group, $\mathrm{P}<0.05$. ${ }^{\mathrm{c} C o m p a r e d}$ with positive control group, $\mathrm{P}<0.05$.
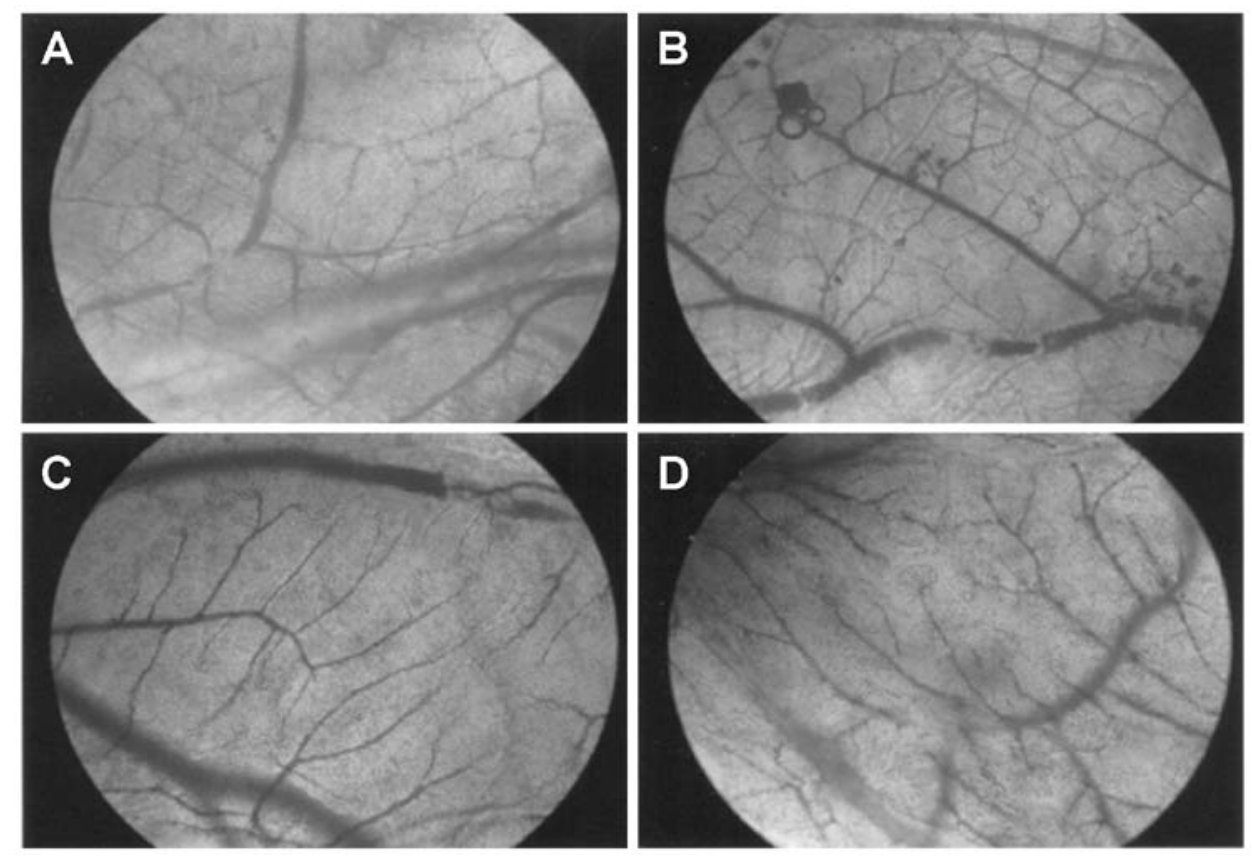

Figure 4. Comparison of vascular network structure in chick embryo chorioallantoic membrane between (A) the blank control group, (B) vascular endothelial growth factor (VEGF)-shRNA group (the vascular branches in the chorioallantoic membrane of the VEGF-shRNA group increased slightly as compared with the blank control group), (C) positive control group (the vascular branches in the chorioallantoic membrane increased more visibly with abnormal capillary network structures present) and (D) negative control group (the vascular branches in the chorioallantoic membrane increased more visibly with abnormal capillary network structures present). Images captured using an optical microscopy (x10 magnification).

Tumor growth rates in nude mouse RCC xenograft model. An RCC xenograft model using nude mice was successfully established with a tumor formation rate of $100 \%$. Ten days following the inoculation of the nude mice with 786-O cells, subcutaneous nodules (approximately $4 \mathrm{~mm}$ ) were observed in the nude mice in the blank control and negative control groups, and the subcutaneous nodules continued to grow over time. Twelve days following subcutaneous injection, subcutaneous nodules gradually appeared in the VEGF-shRNA group. The growth of subcutaneous nodules in the VEGF-shRNA group was slower than that in the blank control and negative control groups, as shown in Table III. The tumor volume in the VEGF-shRNA group was significantly smaller than taht the blank control and negative control groups at 10, 15, 25 and 30 days after subcutaneous injection (all $\mathrm{P}<0.05)$. No detectable significant differences in tumor volume between the blank control and the negative control groups were observed at 10, 15, 25 and 30 days after subcutaneous injection (all $\mathrm{P}>0.05$ ). Tumor growth curves in the blank control, negative control, and VEGF-shRNA groups are presented in Fig. 5. Compared with the blank control and negative control groups, tumor growth in the VEGF-shRNA group was inhibited. Thirty days after subcutaneous injection, all the nude mice were sacrificed, and the RCC xenograft tumors were removed and weighed (Table IV). The weights of the RCC xenograft tumors in the negative control, blank control and VEGF-shRNA groups were $2.204 \pm 0.207,2.239 \pm 0.337$ and $0.663 \pm 0.086 \mathrm{~g}$, respectively. The tumor weight in the VEGF-shRNA group was significantly lower than that the negative control and blank control groups (both $\mathrm{P}<0.05$ ). There was no observable significant difference in tumor weight between the negative control and blank control groups $(\mathrm{P}>0.05)$. 
Table III. Volume of renal cell carcinoma (RCC) xenograft tumors in nude mice in different treatment groups at different time points.

\begin{tabular}{lccc}
\hline Time point & Negative control group & Blank control group & VEGF-shRNA group \\
\hline 5 days & 0 & 0 & 0 \\
10 days & $83.15 \pm 8.63^{\mathrm{a}}$ & $85.14 \pm 8.72^{\mathrm{a}}$ & 0 \\
15 days & $503.42 \pm 17.58^{\mathrm{a}}$ & $519.18 \pm 17.97^{\mathrm{a}}$ & $87.35 \pm 8.52$ \\
20 days & $1267.49 \pm 60.59^{\mathrm{a}}$ & $1303.47 \pm 65.26^{\mathrm{a}}$ & $381.58 \pm 16.18$ \\
25 days & $2324.59 \pm 89.53^{\mathrm{a}}$ & $2409.19 \pm 90.16^{\mathrm{a}}$ & $794.90 \pm 21.59$ \\
30 days & $3880.12 \pm 118.63^{\mathrm{a}}$ & $3944.65 \pm 120.41^{\mathrm{a}}$ & $1211.50 \pm 59.64$ \\
\hline
\end{tabular}

Tumor volume shown in $\mathrm{mm}^{3}$. Negative control group, mice injected subcutaneously in the lower back with $\sim 5 \times 10^{6}$ cells transfected with LV-NCshRNA in $0.2 \mathrm{ml}$ of PBS qod; blank control group, mice injected subcutaneously in the rear back with $\sim 5 \times 10^{6}$ cells without viral transfection in $0.2 \mathrm{ml}$ of PBS qod; VEGF-shRNA group, mice injected subcutaneously in the rear back with $\sim 5 \times 10^{6}$ cells transfected with LV-VEGF-shRNA in

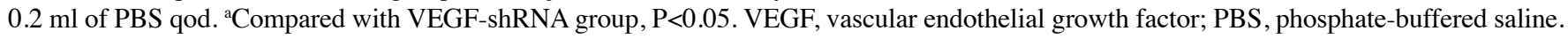

Table IV. Weight of renal cell carcinoma (RCC) xenograft tumors in nude mice in the different treatment groups 30 days after subcutaneous injection.

\begin{tabular}{lc}
\hline Group & Tumor weight $(\mathrm{g})$ \\
\hline Negative control group & $2.204 \pm 0.207^{\mathrm{a}}$ \\
Blank control group & $2.239 \pm 0.337^{\mathrm{a}}$ \\
VEGF shRNA group & $0.663 \pm 0.086$ \\
\hline
\end{tabular}

Negative control group, mice injected subcutaneously in the lower back with $\sim 5 \times 10^{6}$ cells transfected with LV-NC-shRNA in $0.2 \mathrm{ml}$ of PBS qod; blank control group, mice injected subcutaneously in the rear back with $\sim 5 \times 10^{6}$ cells without viral transfection in $0.2 \mathrm{ml}$ of PBS qod; VEGF-shRNA group, mice injected subcutaneously in the rear back with $\sim 5 \times 10^{6}$ cells transfected with LV-VEGF-shRNA in $0.2 \mathrm{ml}$ of PBS qod. ${ }^{\mathrm{a} C o m p a r e d}$ with VEGF-shRNA group, $\mathrm{P}<0.05$. VEGF, vascular endothelial growth factor; PBS, phosphate-buffered saline.

VEGF expression in nude mouse RCC xenograft model. IHC staining detected VEGF expression (brownish yellow) in the xenograft tumors, mainly in the cytoplasm and/or membranes. Slices of IHC-stained RCC xenograft tumors from the VEGF-shRNA, negative control and blank control groups were observed by a double-blind method (Fig. 6). The tumor slices from the VEGF-shRNA group displayed a decreased VEGF expression, specifically in the cytoplasm and membrane. As shown in Fig. 7, VEGF expression in the VEGF-shRNA group was lower than that in the negative control and blank control groups (10.9 vs. 62.5 vs. $67.3 \%$, all $\mathrm{P}<0.05)$. No obvious differences in VEGF expression were detected between the negative control and the blank control groups ( $P>0.05$, Fig. 7). The results of IHC staining suggested that transfection with VEGFshRNA markedly inhibited the protein expression of VEGF in nude mice.

Tumor growth rates in tumor-bearing nude mice. On day 7 after subcutaneousinjection, thetumordiameteronaverage was found to be $\geq 5 \mathrm{~mm}$ in all mice. The nude mice were intratumorally injected with various treatments [LV-VEGF-shRNA (treat-



Figure 5. Tumor growth curves from renal cell carcinoma (RCC) xenograft nude mouse models showing a marked inhibition of tumor growth in the vascular endothelial growth factor (VEGF)-shRNA group compared with the negative control and blank control groups.

ment group), LV-NC-shRNA (negative control group) and mice intratumorally injected with PBS (blank control group)]. Compared with the negative control and blank control groups, there were no significant differences in tumor volume in the VEGF-shRNA treatment group during the first 4 days after treatment (both $\mathrm{P}>0.05$; Fig. 8 and Table V). The treatment group showed a significantly reduced tumor growth on the 8th day after treatment (Fig. 8 and Table V). At 8 days after treatment, the tumor volume in the treatment group was significantly smaller than that the negative control and blank control groups (both $\mathrm{P}<0.05$ ). No obvious significant differences in tumor volume were observed between the negative control and blank control groups 8 days after treatment $(\mathrm{P}>0.05)$.

Apoptosis in tumor-bearing nude mice. Brownish yellow nuclei, chromatin condensation, irregular cell morphology, and light-colored cells were observed in the treatment group (Fig. 9). The blank control and negative control groups showed a regular cell morphology and few light-colored cells. As shown in Fig. 10, the apoptotic index in the treatment group was significantly higher than that in the blank control 
Table V. Comparison of subcutaneous tumor volume in tumor-bearing nude mice in the different treatment groups at different time points.

\begin{tabular}{lcccrr}
\hline Time point & Blank control group & Negative control group & Treatment group & F-value & P-value \\
\hline 1 day & $80.15 \pm 7.96$ & $77.85 \pm 7.13$ & $75.64 \pm 7.09$ & 0.557 & 0.585 \\
4 days & $330.56 \pm 18.23$ & $339.78 \pm 18.94$ & $342.89 \pm 18.52$ & 0.716 & 0.505 \\
8 days & $1341.12 \pm 61.78^{\mathrm{a}}$ & $1278.03 \pm 60.90^{\mathrm{a}}$ & $940.45 \pm 21.58$ & 104.5 & $<0.001$ \\
12 days & $1782.42 \pm 71.51^{\mathrm{a}}$ & $1846.63 \pm 71.71^{\mathrm{a}}$ & $1228.02 \pm 60.15$ & 150.1 & $<0.001$ \\
16 days & $2931.20 \pm 91.04^{\mathrm{a}}$ & $2872.57 \pm 92.55^{\mathrm{a}}$ & $1610.21 \pm 82.10$ & 424.9 & $<0.001$ \\
20 days & $3678.78 \pm 117.14^{\mathrm{a}}$ & $3564.63 \pm 115.16^{\mathrm{a}}$ & $2038.23 \pm 86.58$ & 438.0 & $<0.001$ \\
\hline
\end{tabular}

Blank control group, mice intratumorally injected with $0.1 \mathrm{ml} \mathrm{PBS} /$ mouse/time; negative control group, mice intratumorally injected with $0.1 \mathrm{ml} \mathrm{LV-NC}$-shRNA/mouse/time; treatment group, mice intratumorally injected with $0.1 \mathrm{ml} \mathrm{LV-VEGF-shRNA/mouse/time.}{ }^{\mathrm{a} C o m p a r e d}$ with treatment group, $\mathrm{P}<0.05 ; 1,4,8,12,16$, and 20 days refers to number of days after treatment. VEGF, vascular endothelial growth factor; PBS, phosphate-buffered saline.


Figure 6. Slices of immunohistochemically-stained renal cell carcinoma (RCC) xenograft tumors removed from nude mice. Samples were obtained from the (A) vascular endothelial growth factor (VEGF)-shRNA group, (B) negative control group and (C) blank control group, and they were observed with a doubleblind method under a microscope (x400 magnification), showing that the tumor slices from the VEGF-shRNA group displayed a obviously decreased VEGF expression, specifically in the cytoplasm and membrane, the tumor slices from the negative control group displayed a strong (positive) expression of VEGF in the cytoplasm and membrane, and the tumor slices from the blank control group displayed a strong (positive) expression of VEGF in the cytoplasm and membrane.



Figure 7. Comparison of the vascular endothelial growth factor (VEGF) positive rate (\%) between the blank control group, negative control group and VEGFshRNA group. The VEGF positive rate in the VEGF-shRNA group (10.9\%) was obviously lower than that in the negative control group (62.5\%) and blank control group (67.3\%). ${ }^{*} \mathrm{P}<0.05$.

and negative control groups $(40.97 \pm 5.47$ vs. $11.06 \pm 1.87$ and $10.76 \pm 1.50 \%$, respectively, all $\mathrm{P}<0.05$ ). There were no obvious differences in the apoptotic index between the blank control and the negative control groups $(\mathrm{P}>0.05)$.

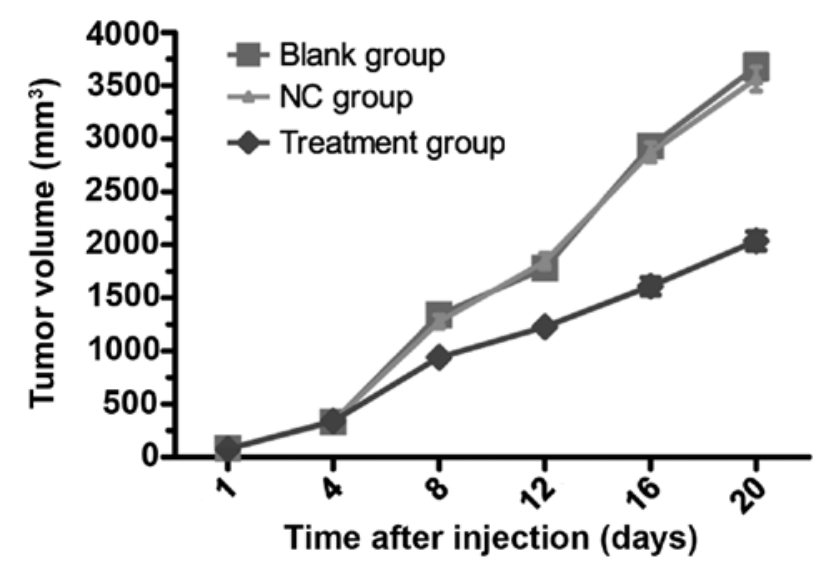

Figure 8. Tumor growth curves in tumor-bearing nude mice treated with intratumoral injection (treatment group, blank control group and negative control group).

\section{Discussion}

At diagnosis, $30-40 \%$ of patients with RCC already suffer from metastatic disease, and radiotherapy or chemotherapy is ineffec- 

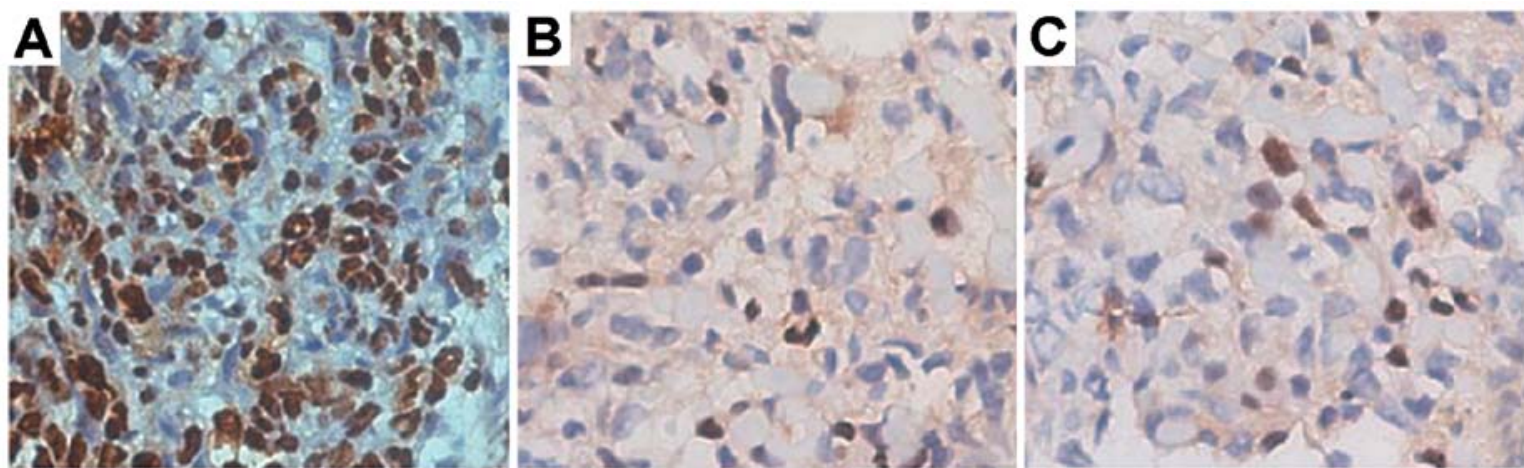

Figure 9. Microscopic observations indicating apoptotic changes associated with cell morphology in tumor tissue removed from tumor-bearing nude mice in the different experimental groups. (A) Vascular endothelial growth factor (VEGF)-shRNA group: brownish-yellow nuclei, chromatin condensation, irregular cell morphology, and light-colored cells were observed; (B) blank control group: regular cell morphology and few light-colored cells were observed; (C) negative control group: regular cell morphology and few light-colored cells were observed.



Figure 10. Comparison of apoptotic index in tumor-bearing nude mice in the treatment group, blank control group and negative control group. A significantly higher apoptotic index was observed in the treatment group.

tive in the majority of cases (16). The most significant promoter of tumor angiogenesis is VEGF (17). The production of high levels of VEGF by solid tumors is a sign of poor prognosis, indicative of rapid disease progression and poor survival (18). This is true for RCC where VEGF is a prominent player in tumor vascularization (19). Therefore, tumor therapy targeting VEGF is likely to be anti-angiogenic and efficacious in RCC (20). Antiangiogenic drug therapy is a novel concept in the treatment of cancer where tumor vasculature is disrupted by the inhibition of molecules/pathways involved in angiogenesis. This causes the tumors to starve due to a lack of blood supply and nourishment, resulting in tumor destruction. This approach has been tested in animal models and appears to have fewer side-effects and lacks drug resistance. Welti et al reported that the vessel sprouting (angiogenesis) model has been studied in the mouse retina, where vascularization occurs post-natally, thus representing a physiological sangiogenesis model (21). In the study by Conley et al, the authors established a tumor-bearing mouse model to determine whether anti-angiogenic agents stimulate an increase in breast cancer stem cells in vivo (22).

In the present study, we developed a recombinant lentiviral LV-VEGF-shRNA tool to silence the expression of VEGF in an RCC cell line. The capability of RNAi to suppress target genes is a promising therapeutic tool against cancer (23). Indeed, transfection of a human RCC cell line with recombinant lentiviral LV-VEGF-shRNA resulted in decreased VEGF expression.
The aim of the present study was to evaluate lentiviral-based RNAi against VEGF as a selective inhibitor of VEGF for the treatment of RCC. Accordingly, we investigated its antitumor and anti-angiogenic functions in a chick embryo chorioallantoic membrane model, a RCC xenograft nude mouse model, and a tumor-bearing nude mouse model, to identify the role of VEGF in vascularization and tumor growth in RCC.

The results obtained from the CAM assay revealed that the relative total vessel length in the chorioallantoic membrane injected with VEGF-shRNA was significantly shorter than that of the controls. This implied that the anti-angiogenic effect of the plenti6.3-miR-200 plasmid was caused by the knockdown of VEGF expression. These findings support the hypothesis that the proliferation of cancer cells is inhibited by the knockdown of VEGF, leading to reduced vessel density in rapid-growing tumors, such as RCC (9).

Equally important results in this study are those achieved by the in vivo subcutaneous injection of a RCC xenograft nude mouse model with 786-O cells transfected with LV-VEGF-shRNA. We found that the growth of subcutaneous nodules in the VEGF-shRNA group was slower than that in the blank control and negative control groups. In addition, tumor volume and tumor weight in the VEGF-shRNA group were significantly lower than those in the controls after subcutaneous injection. These results suggested that tumor growth was obviously inhibited by the knockdown of VEGF and supplied evidence in support of the anti-angiogenic activity of VEGF-shRNA. Antitumor efficacy may be the resulted of the inhibition of tumor angiogenesis as demonstrated by decreased intratumoral microvessel density. In line with our findings, a previous study detected that the transfection of tumor cells with siRNA-VEGF inhibited tumor growth and vessel density in prostate cancer (24).

Considering future therapeutic applications to advanced RCC, athe systemic administration of therapeutic molecules/agents is of utmost interest as RCC progresses rapidly and its metastatic spread is extensive. In this study, we successfully validated the antitumor efficacy of the in vivo delivery of a lentvirus expressing VEGF-shRNA by intratumoral injection in tumor-bearing nude mice. Nude mice receiving VEGF-shRNA showed considerably reduced tumor growth compared to the controls, which further confirmed the antitumor effects 
of VEGF-shRNA in RCC. The antitumor effect of an intratumoral injection with VEGF-shRNA, as described in this study, is consistent with the results of a previously published study which showed that the transfer of siRNA-VEGF led to a $67 \%$ decrease in tumor growth in a fibrosarcoma model (25). Further studies are required to design better systemic delivery methods of lentivirus-based therapeutic agents to allow a more effective antitumor response.

In conclusion, in this study, we demonstrated that treatment with VEGF-shRNA reduced VEGF protein levels in vitro and in vivo, and inhibited vessel formation and tumor growth in RCC. Therefore, treatment with a shRNA expression vector targeted against VEGF may be a powerful tool for the future treatment of RCC.

\section{Acknowledgements}

This study was funded by grants from the National Natural Science Foundation (nos. 81172450, 81202008 and 81402089), the Pudong Health Bureau of Shanghai (no. PW2013D-3) and the Key Disciplines Group Construction Project of the Pudong Health Bureau of Shanghai (PWZxq2014-11). We would like to acknowledge the reviewers for their helpful comments on this study.

\section{References}

1. Sato Y, Yoshizato T, Shiraishi Y, Maekawa S, Okuno Y, Kamura T, Shimamura T, Sato-Otsubo A, Nagae G, Suzuki H, et al: Integrated molecular analysis of clear-cell renal cell carcinoma Nat Genet 45: 860-867, 2013.

2. Koul H, Huh JS, Rove KO, Crompton L, Koul S, Meacham RB and Kim FJ: Molecular aspects of renal cell carcinoma: a review. Am J Cancer Res 1: 240-254, 2011.

3. Lin JA, Fang SU, Su CL, Hsiao CJ, Chang CC, Lin YF and Cheng CW: Silencing glucose-regulated protein 78 induced renal cell carcinoma cell line G1 cell-cycle arrest and resistance to conventional chemotherapy. Urol Oncol 32: 29.e1-11, 2014

4. Molina AM, Tickoo SK, Ishill N, Trinos MJ, Schwartz LH, Patil S, Feldman DR, Reuter VE, Russo P and Motzer RJ: Sarcomatoidvariant renal cell carcinoma: treatment outcome and survival in advanced disease. Am J Clin Oncol 34: 454-459, 2011.

5. Cho IC and Chung J: Current status of targeted therapy for advanced renal cell carcinoma. Korean J Urol 53: 217-228, 2012.

6. Zhang X, Wang W, Mize GJ, Takayama TK, True LD and Vessella RL: Protease-activated receptor 2 signaling upregulates angiogenic growth factors in renal cell carcinoma. Exp Mol Pathol 94: 91-97, 2013.

7. Chen Y, Dawes PT and Mattey DL: Polymorphism in the vascular endothelial growth factor A (VEGFA) gene is associated with serum VEGF-A level and disease activity in rheumatoid arthritis: differential effect of cigarette smoking. Cytokine 58: 390-397, 2012.
8. Larsen AK, Ouaret D, El Ouadrani K and Petitprez A: Targeting EGFR and VEGF(R) pathway cross-talk in tumor survival and angiogenesis. Pharmacol Ther 131: 80-90, 2011.

9. Takahashi S: Vascular endothelial growth factor (VEGF), VEGF receptors and their inhibitors for antiangiogenic tumor therapy. Biol Pharm Bull 34: 1785-1788, 2011.

10. Gray RT, O'Donnell ME, Maxwell P, McGuigan JA and Spence GM: Long-term follow-up of immunocytochemical analysis of vascular endothelial growth factor (VEGF), and its two receptors, VEGF-R1 (Flt-1) and VEGF-R2 (Flk-1/KDR), in oesophagogastric cancer. Int J Biol Markers 28: 63-70, 2013.

11. Wang L, Rahman S, Lin CY, Valdivia J, Than K, La Marca F and Park P: A novel murine model of human renal cell carcinoma spinal metastasis. J Clin Neurosci 19: 881-883, 2012.

12. Albiges L, Salem M, Rini B and Escudier B: Vascular endothelial growth factor-targeted therapies in advanced renal cell carcinoma. Hematol Oncol Clin North Am 25: 813-833, 2011.

13. Castel SE and Martienssen RA: RNA interference in the nucleus: roles for small RNAs in transcription, epigenetics and beyond. Nat Rev Genet 14: 100-112, 2013.

14. Sliva K and Schnierle BS: Selective gene silencing by viral delivery of short hairpin RNA. Virol J 2010, 7: 248, 2010.

15. Benson DA, Boguski M, Lipman DJ and Ostell J: GenBank. Nucleic Acids Res 22: 3441-3444, 1994

16. Siegel R, DeSantis C, Virgo K, Stein K, Mariotto A, Smith T, Cooper D, Gansler T, Lerro C, Fedewa S, et al: Cancer treatment and survivorship statistics, 2012. CA Cancer J Clin 62: 220-241, 2012.

17. Gerber DE: Targeted therapies: a new generation of cancer treatments. Am Fam Physician 77: 311-319, 2008.

18. Eichelberg C, Junker K, Ljungberg B and Moch H: Diagnostic and prognostic molecular markers for renal cell carcinoma: a critical appraisal of the current state of research and clinical applicability. Eur Urol 55: 851-863, 2009.

19. Ellis LM and Hicklin DJ: VEGF-targeted therapy: mechanisms of anti-tumour activity. Nat Rev Cancer 8: 579-591, 2008.

20. Lane HA, Wood JM, McSheehy PM, Allegrini PR, Boulay A, Brueggen J, Littlewood-Evans A, Maira SM, Martiny-Baron G, Schnell CR, et al: mTOR inhibitor RAD001 (everolimus) has antiangiogenic/vascular properties distinct from a VEGFR tyrosine kinase inhibitor. Clin Cancer Res 15: 1612-1622, 2009.

21. Welti J, Loges S, Dimmeler S and Carmeliet P: Recent molecular discoveries in angiogenesis and antiangiogenic therapies in cancer. J Clin Invest 123: 3190-3200, 2013.

22. Conley SJ, Gheordunescu E, Kakarala P, Newman B, Korkaya H, Heath AN, Clouthier SG and Wicha MS: Antiangiogenic agents increase breast cancer stem cells via the generation of tumor hypoxia. Proc Natl Acad Sci USA 109: 2784-2789, 2012.

23. Gandellini P, Profumo V, Folini M and Zaffaroni N: MicroRNAs as new therapeutic targets and tools in cancer. Expert Opin Ther Targets 15: 265-279, 2011.

24. Wannenes F, Ciafré SA, Niola F, Frajese G and Farace MG: Vector-based RNA interference against vascular endothelial growth factor-A significantly limits vascularization and growth of prostate cancer in vivo. Cancer Gene Ther 12: 926-934, 2005.

25. Filleur S, Courtin A, Ait-Si-Ali S, Guglielmi J, Merle C, HarelBellan A, Clézardin P and Cabon F: SiRNA-mediated inhibition of vascular endothelial growth factor severely limits tumor resistance to antiangiogenic thrombospondin-1 and slows tumor vascularization and growth. Cancer Res 63: 3919-3922, 2003. 